Pacific Journal of Mathematic 


\title{
DEFORMING P. L. HOMEOMORPHISMS ON A CONVEX POLYGONAL 2-DISK
}

\author{
ChUnG-WU Ho
}

\begin{abstract}
It is shown that for each $p . l$. homeomorphism $f$ on a convex polygonal disk which is pointwise fixed on the boundary of the disk, there exists a triangulation $K$ of the disk such that $f$ may be obtained by successively moving the vertices of $K$ (with the motion being extended linearly to each triangle of $K$ ) in a finite number of steps such that no triangles will be collapsed in the process of motion. An algebraic interpretation of this result is also given.
\end{abstract}

1. Introduction. By a convex polygonal disk in $R^{2}$, we mean a closed region $D$ in $R^{2}$ enclosed by a convex polygon $P$. The vertices of $P$ will also be called the vertices of the disk $D$. We shall always assume that no three consecutive vertices of $D$ lie on a straight line. By a triangulation of $D$, we mean a (rectilinear) simplicial complex with $D$ as its underlying space. If $K$ is a triangulation of $D$, we shall let $L(K)$ denote the set of all homeomorphisms from $D$ onto $D$ which are linear on each simplex of $K$ and are pointwise fixed on $\mathrm{Bd}(D)$. Elements of $L(K)$ will be called linear homeomorphisms of $D$ with respect to $K$. We shall consider $L(K)$ as a topological space with the compact open topology. A map $f: D \rightarrow D$ will be called a p.l. homeomorphism of $D$ if $f \in L(K)$ for some triangulation $K$ of $D$. A triangulation $K$ of $D$ will be called a proper triangulation if the only 0-simplices of $K$ lying on $\mathrm{Bd}(D)$ are those which are the vertices of $D$. Finally, a p.l. homeomorphism $f$ of $D$ is called proper if $f \in L(K)$ for some proper triangulation $K$ of $D$. In the next section, we shall establish the following:

Theorem A. Let $D$ be a convex polygonal disk in $R^{2}$. For any proper triangulation $K$ of $D$, the space $L(K)$ is pathwise connected.

Note that a path in the space $L(K)$ with the initial point $f$ and terminal point $g$ corresponds to a deformation from $f$ to $g$ through a family of homeomorphisms of $D$ onto $D$ which are linear with respect to $K$ and are pointwise fixed on $\mathrm{Bd}(D)$. When $D$ is a triangle in $R^{2}$, Theorem $\mathrm{A}$ is a consequence of $\mathrm{S}$. $\mathrm{S}$. Cairns' result on the deformation of isomorphically imbedded triangulations in the plane [2], [3] also [4, Proposition 2.19]. However, if $D$ is not a triangle, 
Cairns' technique, unlike ours, will in general carry the deformation outside the disk $D$. Using Theorem A, we shall show in $\S 3$ that a general (i.e., not necessarily proper) $p$. $l$. homeomorphism $f$ of $D$ may still be deformed into the identity map of $D$ (or vice versa) in some space $L(K)$. Finally in $\S 4$, we shall improve our result of $\S 3$ to the following statement, which together with Theorem A form the main results of this paper.

TheOREM B. For each p.l. homeomorphism $f$ of $D$, there exists a triangulation $K$ of $D$ such that $f$ may be obtained in a finite number of steps by successively moving the vertices of $K$ (with the motion being extended linearly to each simplex of $K$ ) such that none of the simplices is collapsed in the process.

The problem of deforming a prescribed map of a space into the identity map, or vice versa, in a specific manner has been studied by many mathematicians. We shall mention some results which are more directly related to the problems considered in this paper. H. Tietze [9] and H. L. Smith [8] showed in 1914 and 1917 respectively that a homeomorphism of a 2-dimensional disk which leaves the boundary pointwise fixed is deformable into the identity map through a family of homeomorphisms which leave the boundary pointwise fixed. 0 . Veblen in 1917 [10] and J. W. Alexander in 1923 [1] extended the result for homeomorphisms of $n$-cells. The technique used by Alexander ("The Alexander Trick") can in fact be used to show that each $p$.l. homeomorphism $f$ on a polyhedral $n$-cell in $R^{n}$ which leaves the boundary fixed can be deformed into the identity map through a family of such $p . l$. homeomorphisms $f_{t}$. However, each of the homeomorphisms $f_{t}$ in this process requires a different triangulation of the domain space. In fact, as $t$ approaches 1 , the triangulation for $f_{t}$ requires triangles which are arbitrarily small. It is therefore natural to ask whether the given p.l. homeomorphism on the polyhedral $n$-cell may be deformed into the identity map through a family of $p . l$. homeomorphisms which are all linear with respect to a fixed triangulation of the $n$-cell. Our Theorem $\mathrm{B}$ clearly answers this question in affirmative for a convex 2-dimensional polyhedral disk. In fact, when the disk is a triangle, we have shown that an entire loop of $p$.l. homeomorphisms of the disk can be deformed into the constant loop at the identity with respect to a fixed triangulation of the disk [5].

2. Deforming proper linear homeomorphisms. In this section, we shall first collect some of the basic properties of the spaces $L(K)$ and then carry out a proof of Theorem A. We shall use a process 
similar to that used by the author in [4] to resolve a special case which will appear in our proof of Theorem A. For this reason, we found it necessary to restate some of the definitions and theorems of [4] in the present framework. The proofs of the theorems will be omitted. In the following, if $K$ is a triangulation of a convex polygonal disk $D$ in $R^{2}$ and if $v$ is a vertex of $K$, we shall let $\operatorname{St}(v, K)$ and $\operatorname{Lk}(v, K)$ to denote respectively the star and link of $v$ with respect to $K$. A vertex $v$ of $K$ will be called an inner vertex of $K$ if $v \notin \mathrm{Bd}(D)$. We shall start with the following observations.

REMARK 2.1. Let $D$ be a convex polygonal disk in $R^{2}$ and $K$ be any triangulation of $D$. For each $f \in L(K)$, the set $\{f(\sigma) \mid \sigma \in K\}$ also forms a triangualtion of $D$. This triangulation will be denoted by $f(K)$. Observe that for each $f \in L(K)$, the spaces $L(K)$ and $L(f(K))$ are homeomorphic; the map: $L(f(K)) \rightarrow L(K)$ carrying each $g \in L(f(K))$ onto $g f$ is clearly a homeomorphism.

DEFINITION 2.2. A triangulation $K$ of a polygonal disk $D$ is said to be decomposable if there are vertices $v_{a}, v_{b}, v_{c}$ of $K$ such that the 1-simplices $\left\langle v_{a}, v_{b}\right\rangle,\left\langle v_{b}, v_{c}\right\rangle$, and $\left\langle v_{c}, v_{a}\right\rangle$ all belong to $K$ but the 2simplex $\left\langle v_{a}, v_{b}, v_{c}\right\rangle$ does not belong to $K$. If $D$ is a triangle, we shall require in addition that at least one of the open simplices $\left(v_{a}, v_{b}\right)$, $\left(v_{b}, v_{c}\right)$, and $\left(v_{c}, v_{a}\right)$ lies in the interior of $D$.

A triangulation $K$ of $D$ is called indecomposable if it is not decomposable.

The following two propositions may be proved essentially the same way as [4, Propositions 1.4 and 1.5].

Proposition 2.3. If $K$ is a decomposable triangulation of a convex polygonal disk $D$ in $R^{2}$, the space $L(K)$ is homeomorphic to a Cartesian product $L\left(K_{1}\right) \times L\left(K_{2}\right)$ where $K_{1}$ is a triangulation of $D$ and $K_{2}$ is a triangulation of a triangle in $R^{2}$ such that each of $K_{1}$ and $K_{2}$ has a fewer number of vertices than $K$.

Proposition 2.4. Let $K$ be a proper, indecomposable triangulation of a convex polygonal disk $D$ in $R^{2}$. For each inner vertex $v_{0}$ of $K$, there is an $f \in L(K)$ such that $\operatorname{St}\left(f\left(v_{0}\right), f(K)\right)$ is strictly convex (i.e., $S t\left(f\left(v_{0}\right), f(k)\right)$ is convex and no three consecutive vertices of $L k\left(f\left(v_{0}\right), f(K)\right)$ lie on a straight line).

Let $K$ be a triangulation of a convex polygonal disk $D$ in $R^{2}$. To study the topological problems of the space $L(K)$, it turns out to be convenient to study some nice subspaces of it. We shall now describe these nice subspaces of $L(K)$. 
Definition 2.5. Let $P$ be a polygonal circle in $R^{2}$ (i.e., $P$ is a simplicial complex in $R^{2}$ with $|P|$ homeomorphic to the 1-sphere $S^{1}$ ). Let $[P]$ be the union of $|P|$ with the bounded component of $R^{2}-|P|$. For each 1-simplex $\left\langle v_{1}, v_{2}\right\rangle$ of $P$, we shall let $H_{P}\left\langle v_{1}, v_{2}\right\rangle$ to denote the open half-plane of $R^{2}$ such that:

1. $\left\langle v_{1}, v_{2}\right\rangle$ lies on $\mathrm{Bd}\left(H_{P}\left\langle v_{1}, v_{2}\right\rangle\right)$.

2. $\bar{H}_{P}\left\langle v_{1}, v_{2}\right\rangle$ (the closed half-plane) contains a neighborhood of Int $\left\langle v_{1}, v_{2}\right\rangle$ in $[P]$.

Now, for each polygonal circle $P$ in $R^{2}$, we define the core of $P$, cor $(P)$, to be the set

$$
\operatorname{cor}(P)=\bigcap\left\{H_{P}\left\langle v_{i}, v_{j}\right\rangle \mid\left\langle v_{i}, v_{j}\right\rangle \text { is a 1-simplex of } P\right\} .
$$

REMARK 2.6. Let $K$ be a triangulation of a convex polygonal disk $D$ in $R^{2}$. Let $v_{0}$ be an inner vertex of $K$. Observe that for each $f \in L(K)$, the vertex $f\left(v_{0}\right)$ must lie in cor $\left(L k\left(f\left(v_{0}\right), f(K)\right)\right)$. Conversely, for each point $x \in \operatorname{cor}\left(\operatorname{Lk}\left(f\left(v_{0}\right), f(K)\right)\right)$, there is a unique element $g \in L(K)$ such that $g(v)=f(v)$ for each vertex $v \neq v_{0}$ of $K$ and $g\left(v_{0}\right)=x$.

DEFINITION 2.7. Let $K$ be a triangulation of a convex polygonal disk $D$. For each inner vertex $v_{i}$ of $K$, we shall let $L_{v_{i}}(K)$ or simply $L_{i}(K)$ denote the subspace of $L(K)$ consisting of all elements $f \in L(K)$ such that $f\left(v_{i}\right)$ is located at the centroid of the set cor $\left(\operatorname{Lk}\left(f\left(v_{i}\right)\right.\right.$, $f(K))$ ).

The space $L_{i}(K)$ is clearly of the same homotopy type as $L(K)$ for each inner vertex $v_{i}$ of $K$ (cf. [4, Proposition 2.6]). The reason for introducing these subspaces $L_{i}(K)$ is that under suitable conditions, they may be decomposed into a finite union of the spaces $L\left(K_{i}\right)$ where each $K_{i}$ is a triangulation of the disk $D$ with one fewer inner vertex than $K$. This makes it possible to carry out an induction argument on the number of vertices of $K$ to prove the pathwise connectedness of $L(K)$. We shall now describe conditions which make such a decomposition of $L_{i}(K)$ possible.

DEFINITION 2.8. Let $K$ be a triangulation of a convex polygonal disk $D$. For each vertex $v_{i}$ of $K$, the incidence number of $v_{i}$ in $K$, denoted by $m_{v_{i}}$ or simply $m_{i}$, is defined as the number of vertices of $K$ lying on $L k\left(v_{i}, K\right)$ (hence, the number of 1-simplices of $K$ incident on $v_{i}$ ).

Proposition 2.9. Let $K$ be a triangulation of a convex polygonal disk $D$ with $n$ inner vertices. Let $v_{0}$ be an inner vertex of $K$ such that 
1. The incidence number $m_{0}$ of $v_{0}$ is $\leqq 5$.

2. $S t\left(v_{0}, K\right)$ is strictly convex.

Then the space $L_{0}(K)$ may be written as the union of at most five subspaces $\left\{L\left(K_{i}\right)\right\}$ where each $K_{i}$ is a triangulation of $D$ with exactly $n-1$ inner vertices. Furthermore, if $K$ is a proper triangulation of $D$, each of the $K_{i}$ 's is also a proper triangulation.

The proof of this proposition, though needing more work to establish, is still similar to that given for [4, Proposition 2.16], hence, will be omitted. Finally, we shall quote a combinatorial lemma [4, Proposition 2.17] which will be used to establish the existence of an inner vertex $v_{0}$ with the incidence number $\leqq 5$ when the triangulation is sufficiently nice.

Proposition 2.10. Let $K$ be a triangulation of a polygonal disk $D$ in $R^{2}$. Then

$$
\sum_{v_{i}=\text { inner vertex of } D}\left(6-m_{i}\right)=6+\sum_{v_{i} \in \operatorname{Bd}(D)}\left(m_{i}-4\right) .
$$

REMARK 2.11. Let $K$ be a triangulation of a polygonal disk $D$ in $R^{2}$ with at least one inner vertex. It follows immediately from Proposition 2.10 that if $D$ is a triangle or if $D$ is a general polygonal disk with $m_{i} \geqq 4$ for each vertex $v_{i} \in \mathrm{Bd}(D)$, then there is at least one inner vertex $v_{0}$ of $K$ with $m_{0} \leqq 5$.

We can now prove Theorem A by induction on the number of vertices of the triangulation $K$. The theorem is clearly true if the number of vertices of $K$ is equal to three, since in that case, $D$ must be a triangle and $K$ has no inner vertex. Hence, $L(K)$ consists only of a single element. Assuming the theorem to be true for any convex polygonal disk in $R^{2}$ with a proper triangulation consisting of less than $n$ vertices, we now consider a convex polygonal disk in $D$ in $R^{2}$ and a proper triangulation $K$ of $D$ of $n$ vertices. We assume that $K$ has at least one inner vertex, for otherwise the theorem is trivially true. By Proposition 2.3, we may also assume that $K$ is an indecomposable triangulation.

We now consider the following special case: For each vertex $v_{i}$ of $K$ lying on $\mathrm{Bd}(D)$, the incidence number $m_{i} \geqq 4$. The theorem may be proved very easily for this case as follows: By Remark 2.11, there is at least one inner vertex $v_{0}$ of $K$ with $m_{0} \leqq 5$. Using Proposition 2.4 and then Remark 2.1 if necessary, we may assume that St $\left(v_{0}, K\right)$ is strictly convex. Applying Proposition 2.9, we see that the space $L_{0}(K)$, which is of the same homotopy type as $L(K)$, may be written as a finite union of the spaces $L\left(K_{i}\right)$, where each $K_{i}$ is a triangulation of $D$ with one fewer vertex than $K$. We may threfore 
apply the induction hypothesis to conclude that each $L\left(K_{i}\right)$ is pathwise connected. Since the identity map of the disk $D$ clearly belongs to all the $L\left(K_{i}\right)$ 's, these pathwise connected spaces $L\left(K_{i}\right)$ have a nonempty intersection. Therefore, $L(K)$ is pathwise connected. With the above special case being taken care of, we need only consider the following case in our inductive step:

Prove that space $L(K)$ is pathwise connected provided that $K$ has a vertex $v_{0} \in \mathrm{Bd}(D)$ with $m_{0} \leqq 3$. Here, as before, $K$ is a proper, indecomposable triangulation of a convex polygonal disk $D$ in $R^{2}$, and has $n(>3)$ vertices.

For any vertex on $\mathrm{Bd}(D)$, the incidence number is clearly at least 2. We first show that the case for $m_{0}=2$ is trivial. Let $v_{1}, v_{2}$ be the two vertices adjacent to $v_{0}$ in either sense along $\mathrm{Bd}(D)$. The fact that $m_{0}=2$ implies that the triangle $\left\langle v_{0}, v_{1}, v_{2}\right\rangle$ is a 2-simplex of $K$. Since $v_{0}, v_{1}, v_{2}$ are all on $\mathrm{Bd}(D)$, each $f \in L(K)$ must be pointwise fixed on this triangle $\left\langle v_{0}, v_{1}, v_{2}\right\rangle$. Hence, the space $L(K)$ is homeomorphic to a space $L\left(K^{\prime}\right)$ where $K^{\prime}$ is the triangulation inherited from $K$ on a smaller convex disk $D^{\prime}$ obtained from $D$ by cutting off the triangle $\left\langle v_{0}, v_{1}, v_{2}\right\rangle$. Our induction hypothesis then guarantees the pathwise connectedness of $L\left(K^{\prime}\right)$, and hence, of $L(K)$.

Henceforth, we may assume that $m_{0}=3$. Again let $v_{1}, v_{2}$ be the two vertices adjacent to $v_{0}$ in either sense along $\mathrm{Bd}(D)$. Let $v_{3}$ be the third vertex such that $\left\langle v_{0}, v_{3}\right\rangle$ is a 1-simplex of $K$. Note that we may assume $v_{3}$ to be an inner vertex. For otherwise, the 1-simplex $\left\langle v_{0}, v_{3}\right\rangle$ would cut the disk into two convex disks $D_{1}, D_{2}$, hence, the space $L(K)$ would be homeomorphic to the Cartesian product $L\left(K_{1}\right) \times$ $L\left(K_{2}\right)$ where $K_{i}$ is the triangulation inherited from $K$ on the disk $D_{i}(i=$ $1,2)$. Pathwise connectedness of $L(K)$ would then be immediate from the induction hypothesis applied to $L\left(K_{1}\right)$ and $L\left(K_{2}\right)$.

Now by Proposition 2.4 and then by Remark 2.1 if necessary, we may assume that $\mathrm{St}\left(v_{3}, K\right)$ is strictly convex. Let an arbitrary $f \in L(K)$ be given. We wish to show that $f$ may be connected to the identity map by a path in the space $L(K)$. We shall consider three cases.

Case 1. The vertex $f\left(v_{3}\right)$ lies in the open triangular region $\left\langle v_{0}, v_{1}, v_{2}\right\rangle$. In this case, we shall first consider a map $g$ defined by $g(v)=v$ for each vertex $v \neq v_{3}$ of $K$ and $g\left(v_{3}\right)=f\left(v_{3}\right)$. Since St $\left(v_{3}, K\right)$ is convex and $f\left(v_{3}\right)$ lies in $\operatorname{St}\left(v_{3}, K\right), g$ is a well-defined element in $L(K)$. Note that $g$ agrees with the identity map outside the region St $\left(v_{3}, K\right)$ in $D$ and $g$ agrees with $f$ on the triangles $\left\langle v_{0}\right.$, $\left.v_{1}, v_{3}\right\rangle$ and $\left\langle v_{0}, v_{2}, v_{3}\right\rangle$. Also note that the identity map may be connected to $g$ by a straight line homotopy which moves the vertex $v_{3}$ to the point $f\left(v_{3}\right)$ along a straight line and keeps all the other 
vertices of $K$ fixed. Let $\gamma$ be the path in $L(K)$ corresponding to this homotopy. $\gamma$ is a path from the identity map to $g$. Observe that $f \circ g^{-1}$ is an element in the space $L(g(K))$ which is pointwise fixed on the triangles $\left\langle g\left(v_{0}\right), g\left(v_{1}\right), g\left(v_{3}\right)\right\rangle$ and $\left\langle g\left(v_{0}\right), g\left(v_{2}\right), g\left(v_{3}\right)\right\rangle$. We may therefore view $f \circ g^{-1}$ as a map on the smaller disk $D^{\prime}$ obtained from $D$ by cutting off the triangles $\left\langle g\left(v_{0}\right), g\left(v_{1}\right), g\left(v_{3}\right)\right\rangle$ and $\left\langle g\left(v_{0}\right), g\left(v_{2}\right)\right.$, $\left.g\left(v_{3}\right)\right\rangle$. The map $f \circ g^{-1}$ is clearly linear with respect to the triangulation $K^{\prime}$ on $D^{\prime}$ inherited from the triangulation $g(K)$ of $D$. The fact that $g\left(v_{3}\right)=f\left(v_{3}\right)$ lies in the interior of the triangular region $\left\langle v_{0}, v_{1}, v_{2}\right\rangle$ implies that the disk $D^{\prime}$ is still strictly convex. Since the triangulation $K^{\prime}$ has a fewer number of vertices than $K\left(v_{0}\right.$ belongs to $K$ but not $K^{\prime}$ ), we may apply the induction hypothesis on the space $L\left(K^{\prime}\right)$ to get a path $\tau^{\prime}$ from the identity map of $D^{\prime}$ to the map $f \circ g^{-1} \mid D^{\prime}$. This path $\tau^{\prime}$ then gives rise to a path $\tau$ in the space $L(g(K))$ from the identity map to the map $f \circ g^{-1}$. Note that $\tau \circ g$ is then a path in the space $L(K)$ from the map $g$ to the map $f$. We may then connect the identity map to the map $f$ in the space $L(K)$ by the path $\gamma$ followed by $\tau \circ g$.

Case 2. The vertex $f\left(v_{3}\right)$ lies on the line segment $\left\langle v_{1}, v_{2}\right\rangle$. In $\S 4$ (Corollary 4.3), we shall prove that the vertex $f\left(v_{3}\right)$ may be moved slightly off the line segment $\left\langle v_{1}, v_{2}\right\rangle$ to produce a path $\gamma_{1}$ in $L(K)$ connecting the element $f$ with an element $f^{\prime} \in L(K)$ where $f^{\prime}(v)=$ $f(v)$ for each $v \neq v_{3}$ in $K$ and $f^{\prime}\left(v_{3}\right)$ lies inside the triangular region $\left\langle v_{0}, v_{1}, v_{2}\right\rangle$. Then by Case $1, f^{\prime}$ may be connected to the identity element of $L(K)$ by a path $\gamma_{2}$. Then $\gamma_{1}$ followed by $\gamma_{2}$ will connect $f$ to the identity element.

Case 3. The vertex $f\left(v_{3}\right)$ lies outside the closed triangular region $\left\langle v_{0}, v_{1}, v_{2}\right\rangle$. By assumption, $\operatorname{St}\left(v_{3}, K\right)$ is a convex open set in the plane. It therefore contains a neighborhood of the open line segment $\left(v_{1}, v_{2}\right)$, and hence, contains points outside the triangle $\left\langle v_{0}, v_{1}, v_{2}\right\rangle$. Moving the vertex $v_{3}$ to any such point if necessary, we may assume that the vertex $v_{3}$ is outside the triangular region $\left\langle v_{0}, v_{1}, v_{2}\right\rangle$.

Now, let $M$ denote the set of all maps in $L(K)$ which carry $v_{3}$ outside the triangular region $\left\langle v_{0}, v_{1}, v_{2}\right\rangle$. By the above assumptions, both $f$ and the identity element of $L(K)$ are in $M$. Therefore, it suffices to prove that $M$ is pathwise connected. We shall do this by showing that $M$ is the homeomorphic image of a map $j$ from some pathwise connected space into the space $L(K)$. Such a pathwise connected space and map $j$ are defined as follows: Let $D^{\prime}$ be the polygonal disk obtained from $D$ by cutting off the triangle $\left\langle v_{0}, v_{1}, v_{2}\right\rangle$. Note that $D^{\prime}$ is still a convex polygonal disk. We now describe a triangulation $K^{\prime}$ of $D^{\prime}$. Outside the triangular region $\left\langle v_{1}, v_{2}, v_{3}\right\rangle$, we 
let $K^{\prime}$ be the same as the triangulation $K$. While on that triangular region, we let $\left\langle v_{1}, v_{2}, v_{3}\right\rangle$ be a 2-simplex of $K^{\prime}$. Note that this determines a proper triangulation $K^{\prime}$ of $D^{\prime}$ which has one fewer vertex $K$ (for $v_{0}$ belongs to $K$ but not to $K^{\prime}$ ). By the induction hypothesis, $L\left(K^{\prime}\right)$ is pathwise connected. We now describe a map $j: L\left(K^{\prime}\right) \rightarrow L(K)$. Consider $D^{\prime}$ as a subset of $D$. Since the disk $D$ is convex, for each element $g \in L\left(K^{\prime}\right)$, observe that the open line segment $\left(g\left(v_{0}\right), g\left(v_{3}\right)\right)$ lies completely in the quadrilateral $\left[v_{0}, v_{1}, g\left(v_{3}\right), v_{2}\right]$. We may therefore define an element $f \in L(K)$ by $f(v)=g(v)$ for all vertices $v \in K^{\prime}$ and $f\left(v_{0}\right)=v_{0}$ (i.e., $f$, as a map on the whole disk $D$, is obtained from $g$ by deleting the 1-simplex $\left\langle v_{1}, v_{2}\right\rangle$ from both $K^{\prime}$ and the image $g\left(K^{\prime}\right)$, and then inserting the 1-simplex $\left\langle v_{0}, v_{3}\right\rangle$ to the domain space and the 1-simplex $\left\langle v_{0}, g\left(v_{3}\right)\right\rangle$ to the image). The map $f$ is a well-defined element in $L(K)$. We let $f=j(g)$. Since for each vertex $v$ of $K^{\prime}$ and for any two elements $g_{1}, g_{2} \in L\left(K^{\prime}\right)$, dist $\left(g_{1}(v), g_{2}(v)\right)=\operatorname{dist}\left(j\left(g_{1}\right)(v), j\left(g_{2}\right)(v)\right)$, the map $j$ is continuous (in fact, it is an isometry). Also note that for each $g \in L\left(K^{\prime}\right)$, the vertex $j(g)\left(v_{3}\right)$ is outside the triangular region $\left\langle v_{0}, v_{1}, v_{2}\right\rangle$. Hence, $j(g)$ belongs to the subset $M$. Conversely, we may easily show that each element $f \in M$ is the image $j(g)$ of some $g \in L\left(K^{\prime}\right)$. This shows that $M$ is the homeomorphic image of the pathwise connected space $L\left(K^{\prime}\right)$ under the map $j . \quad M$ must be pathwise connected. This finishes the proof of Theorem A.

3. Deforming arbitrary p.1. homeomorphisms. In this section, we shall show that each arbitrary p.l. homeomorphism on a convex polygonal disk $D$ is deformable into the identity map in the space $L(K)$ for some triangulation $K$ of $D$. We do this by first showing that each p.l. homeomorphism on $D$ is deformable into a proper one. The deformation process may then be completed by using Theorem A. We start with a trivial observation which will be needed in the deformation process.

Lemma 3.1. Let $D$ be a convex polygonal disk in $R^{2}$ and $K$ be an arbitrary triangulation of $D$. For each subdivision $K^{\prime}$ of $K$, the space $L(K)$ may be considered in a natural way as a subspace of the space $L\left(K^{\prime}\right)$.

Proof. Observe that each element $f$ in $L(K)$ is also linear with respect to the triangulation $K^{\prime}$, and hence, may be considered as an element of $L\left(K^{\prime}\right)$.

Proposition 3.2. Let $K$ be an arbitrary triangulation of a convex polygonal disk $D$ in $R^{2}$. For each element $f \in L(K)$, there exist triangulations $K_{1}, K_{2}$ of $D$ such that 
1. $K_{1}$ is a proper triangulation of $D . \quad K_{2}$ is a common subdivision of $K$ and $K_{1}$. (Hence, $L\left(K_{1}\right)$ and $L(K)$ may both be considered as subspaces of $L\left(K_{2}\right)$.)

2. The element $f$ may be deformed in $L\left(K_{2}\right)$ into an element $f^{\prime}$ contained in $L\left(K_{1}\right)$.

Outline of the proof. When $D$ is a triangle, this theorem is proved in detail in [5, Theorem 1.3]. In fact, we showed there how to deform, instead of a single element $f$ in $L(K)$, a compact set of elements in $L(K)$. The same proof, with obvious modifications, also works here. We shall therefore only give a brief outline of the proof.

For a sufficiently small positive number $\delta$, we let $D_{\delta}$ be the disk lying inside $D$ and concentric to $D$ (for some point in Int $(D)$ ) such that the perpendicular distances between the corresponding parallel sides of $D$ and $D_{\delta}$ are $\delta$. We also let $B_{\delta}$ be the annular strip Cl ( $D$ $D_{\delta}$ ) where $\mathrm{Cl}(X)$ means the closure of $X$ with respect to $D$. Each $D_{\delta}$ gives rise to a decomposition of $D$ into a rectilinear cell complex (in the sense of $[7, \mathrm{p} .74]$ or $[6, \mathrm{p} .5]$ ) which is obtained by letting the disk $D_{\hat{o}}$ be a 2-cell and by cutting the region $B_{o}$ into 2-cells by connecting each vertex of $D$ to the closest vertex of $D_{\dot{\delta}}$ by a 1-cell. We denote this cell complex by $R(\delta)$.

Let $K$ be an arbitrary triangulation of the disk $D$ and consider an arbitrary element $f \in L(K)$. If $K$ is proper, we simply let $K_{1}$ be $K$ and $f^{\prime}$ be $f$ and there is nothing to prove. Hence, we may assume that $K$ is not proper. We first fix a number $\alpha>0$ such that

1. All the inner vertices of $K$ are contained in $\operatorname{Int}\left(D_{\alpha}\right)$.

2. If $q$ is an inner 1 -simplex of $K$ with both vertices on $\operatorname{Bd}(D)$, then $q \cap \operatorname{Int}\left(D_{\alpha}\right) \neq \varnothing$.

We then fix a sufficiently small number $\delta(0<\delta<\alpha)$ and let $J(\delta)$ be the rectilinear cell complex obtained by imposing the two cell complexes $R(\alpha)$ and $R(\delta)$ on $K$ (i.e., each cell in $J(\delta)$ is an intersection of cells of $R(\alpha), R(\delta)$, and $K)$. Finally, we let $K(\delta)$ be a simplicial subdivision of $J(\delta)$ without adding any more vertices (i.e., we get $K(\delta)$ by adding a number of diagonals to each 2-cell of $J(\delta)$ which is not a triangle). $K(\delta)$ is then a simplicial subdivision of $K$, hence, $f \in L(K(\delta))$.

The idea of the proof is this. We first deform the element $f$ in the space $L(K(\delta))$ into an element $f^{\prime}$ such that $f^{\prime} \mid B_{o}$ is the identity map of $B_{\dot{j}}$. We then observe that any such $f^{\prime}$ may be considered as a linear homeomorphism with respect to a proper triangulation $K_{1}$ of $D$. If we let $K_{2}$ be a common simplicial subdivision of $K_{1}$ and $K(\delta)$, the triangulations $K_{1}$ and $K_{2}$ will clearly satisfy all the conditions of the proposition.

A deformation $F$ carrying $f$ into an $f^{\prime}$ described above can be 
defined as follows: For each vertex $v$ of $K(\delta)$ which does not lie on the polygon $\mathrm{Bd}\left(D_{\delta}\right)$, we let $F(v, t)=f(v)$ for all $t$ (i.e., we fix the image of $f$ on the set $f\left(D_{\alpha}\right)$ and on $\left.\mathrm{Bd}(D)\right)$. For all the vertices $v$ 's of $K(\delta)$ which lie on $\mathrm{Bd}\left(D_{\delta}\right)$, we pull all the $f(v)$ 's simultaneously back to the $v$ 's. It can be shown that if $\delta$ is sufficiently small (e.g., $\delta$ satisfies the conditions given in [5, Definition 3.5]) this will give us a deformation in the space $K(\delta)$. The resulting map $f^{\prime}$ then agrees with $f$ on the set $f\left(D_{\alpha}\right)$ and $f^{\prime} \mid B_{\delta}=$ identity map. The reader is referred to [5] for details.

We then observe that $f^{\prime}$ may be considered as an element in $L\left(K_{1}\right)$ for some proper triangulation $K_{1}$ of $D$. We now describe such a proper triangulation. We let $K_{1}$ be identical with $K(\delta)$ on the closed region $D_{\hat{\delta}}$ of $D$. While on the annular strip $B_{\delta}$, we firrst cut the strip into a number of 2-cells by the cell decomposition $R(\delta)$ on this strip (each of these 2-cells will then be a quadrilateral). We then triangulate the strip by cutting each of these 2-cells into triangles in the following manner: Pick a vertex $v$ of the 2-cell which is also a vertex of $D$. Note that exactly one of the sides of this 2-cell lies in the polygon $\mathrm{Bd}\left(D_{\delta}\right)$. Let $v_{1}, v_{2}, \cdots, v_{k}$ be vertices of $K(\delta)$ lying on this side of the 2-cell. We shall then cut the 2-cell into triangles by inserting a diagonal from $v$ to each vertex $v_{i}$ for $i=1,2, \cdots, k$. This gives us a desired proper triangulation $K_{1}$.

We contend that the map $f^{\prime}$ belongs to $K_{1}$. Observe that $f^{\prime}$ is linear on each simplex $\sigma$ of $K_{1}$ lying in $D_{\delta}$, for $\sigma$ is also a simplex of $K(\delta) . \quad f^{\prime}$ is also linear on each simplex $\delta$ of $K_{1}$ lying on $B_{\delta}$, for $f^{\prime}$ is the identity map there. Hence $f^{\prime} \in L\left(K_{1}\right)$. The proposition then follows by letting $K_{2}$ be a common simplicial subdivision of $K_{1}$ and $K(\delta)$.

THEOREM 3.3. Let $K$ be an arbitrary triangulation of a convex polygonal disk $D$ in $R^{2}$. For each linear homeomorphism $f \in L(K)$, there exists a simplicial subdivision $K^{\prime}$ of $K$ and path $\gamma: I \rightarrow L\left(K^{\prime}\right)$ such that $\gamma(0)=f$ and $\gamma(1)=$ the identity map of $D$.

Proof. Consider any triangulation $K$ and any $f \in L(K)$. By Proposition 3.2, we may find a proper triangulation $K_{1}$ of $D$ and a triangulation $K_{2}$ which is a common subdivision of $K$ and $K_{1}$ such that there is a path $\gamma_{1}$ in $L\left(K_{2}\right)$ connecting $f$ to some element $f^{\prime}$ of $L\left(K_{1}\right)$. By Theorem A, we may also get a path $\gamma_{2}$ in the space $L\left(K_{1}\right)$ from the element $f^{\prime}$ to the identity map of $D$. Since $K_{2}$ is a subdivision of $K_{1}$, the path $\gamma_{2}$ is also a path in the space $L\left(K_{2}\right)$. We may therefore set $K^{\prime}=K_{2}$. Using the path $\gamma_{1}$ followed by $\gamma_{2}$, we get a path in the space $L\left(K^{\prime}\right)$ connecting $f$ to the identity map of $D$. 
COROLLARY 3.4. Each p.l. homeomorphism of a convex polygonal disk $D$ in $R^{2}$ is deformable to the identity map of $D$ in the space $L(K)$ for some triangulation $K$ of $D$.

4. Decomposing a deformation into a finite sequence of single moves. In this section, we shall prove our Theorem B of $\S 1$. Let $D$ be a convex polygonal disk $D$ in $R^{2}$. We first make some observations on the topology of the space $L(K)$ for an arbitrary triangulation $K$ of $D$.

REMARK 4.1. For each triangulation $K$ of $D$, observe that the space $L(K)$ is metrizable, say with a metric $\gamma(f, g)=\max _{v \in S}\{d(f(v)$, $g(v))\}$, where $S$ is the set of inner vertices of $K$ and $d$ is the supmetric of the Euclidean plane i.e., $d\left(\left(x_{1}, x_{2}\right),\left(y_{1}, y_{2}\right)\right)=\max _{i=1,2}\left|x_{i}-y_{i}\right|$.

Proposition 4.2. Let $K$ be any triangulation of $D$ with $k$ inner vertices. The space $L(K)$ may be identified with an open subset of $R^{2} \times R^{2} \times \cdots \times R^{2}$ (k copies).

Proof. In the following, all the maps from $D$ into $R^{2}$ are assumed to be pointwise fixed on $\mathrm{Bd}(D)$. By a linear map $f: K \rightarrow R^{2}$, we mean a map $f: D \rightarrow R^{2}$ (not necessarily a homeomorphism into) which is linear with respect to each simplex of $K$. First observe that each linear map from $K$ into $R^{2}$ is completely determined by its images of the inner vertices of $K$. Suppose that an ordering is assigned to the inner vertices, say $v_{1}, v_{2}, \cdots, v_{k}$ of $K$. The correspondence between a linear map $f: K \rightarrow D$ and the point $\left(f\left(v_{1}\right), f\left(v_{2}\right), \cdots, f\left(v_{k}\right)\right) \in R^{2} \times$ $R^{2} \times \cdots \times R^{2}$ ( $k$ copies) gives rise to a one-to-one correspondence between the set of all such linear maps and $R^{2 k}$. Under this correspondence, the space $L(K)$ is identified with la subset of $R^{2 k}$. This identification is indeed a homeomorphism of $L(K)$ into $R^{2 k}$. In fact, it is an isometry into $R^{2 k}$ if $L(K)$ is given the metric $\gamma$ and $R^{2 k}$ is given the sup-metric of the Euclidean space.

To see that the image of $L(K)$ in $R^{2 k}$ is an open subset, we need only show that any linear map $g$ from $K$ to $R^{2}$ belongs to $L(K)$ if it is sufficiently close to some element of $L(K)$ under the metric $\gamma$. This can be done by means of a result of J.H. C. Whitehead, that a sufficiently close $C^{1}$ approximation to an immersion from a complex into a manifold is itself an immersion [11] (also see [7, Theorem 8.8]). However, we shall sketch a more elementary argument here. Observe that a linear map $g$ from $K$ into $R^{2}$ belongs to $L(K)$ if and only if the image under $g$ of each 2 -simplex has positive area (i.e., nonzero, nonnegative area) (cf. 5, Lemma 2.1]). If $g$ has this property, any linear map: $K \rightarrow K^{2}$ sufficiently close to $g$ must also have this property. 
This shows that $L(K)$ must correspond to an open subset of $R^{2 k}$. This finishes the proof.

The following corollary was needed in $\S 1$. It follows immediately from the Proposition 4.2 and the fact that Euclidean spaces are locally pathwise connected.

Corollary 4.3. For each triangulation $K$ of $D$, the space $L(K)$ is locally pathwise connected. In fact, for each $f \in L(K)$, there exists a number $\delta>0$ such that for each linear map $g: K \rightarrow R^{2}$ with $g \mid \mathrm{Bd}(D)=$ identity and $d(g(v), f(v))<\delta$ for all inner vertices $v$ of $K$, the map $g$ belongs to $L(K)$ and may be connected to $f$ by a path in $L(K)$.

Proof of Theorem B. Consider any p.l. homeomorphism $f$ of $D$. By Corollary 3.4, there exists a triangulation $K$ of $D$ and a path $\gamma: I \rightarrow L(K)$ such that $\gamma$ connects $f$ to the identity map of $D$. Let $k$ be the number of inner vertices of $K$. Using Proposition 4.2, we may find, for each point $x \in \gamma(I)$ an open rectangular box $U_{x}=V_{1} \times$ $V_{2} \times \cdots \times V_{k}$ of $R^{2 k}$ containing $x$ such that $U_{x} \subset L(K)$ and each $V_{i}$ is an open rectangle in $R^{2}$. Let $\varepsilon$ be the Lebesegue number of the open covering $\left\{\gamma^{-1}\left(U_{x}\right) \mid x \in \gamma(I)\right\}$ of the unit interval $I$ and let $0=$ $t_{0}<t_{1}<\cdots<t_{n}=1$ be a partition of the unit interval such that the length of each closed interval $\left[t_{i-1}, t_{i}\right]$ is less than $\varepsilon$.

We now show that for each $i=1, \cdots, n$, the element $\gamma\left(t_{\imath-1}\right)$ may be deformed into the element $\gamma\left(t_{i}\right)$ in the space $L(K)$ in a finite number of steps by succesively moving the vertices of $\gamma\left(t_{i-1}\right)$. Consider any particular $i(1 \leqq i \leqq n)$. Let $\gamma\left(t_{i-1}\right)=\left(a_{1}, a_{2}, \cdots, a_{k}\right) \in R^{2 k}$ and $\gamma\left(t_{i}\right)=$ $\left(b_{1}, b_{2}, \cdots, b_{k}\right) \in R^{2 k}$. We may choose an open rectangular box $U=$ $V_{1} \times V_{2} \times \cdots \times V_{k}$ in $L(K)\left(\subset R^{2 k}\right)$ containing both $\gamma\left(t_{r-1}\right)$ and $\gamma\left(t_{i}\right)$. Since the whole rectangular box $U$ is contained in $L(K)$, we may move $\gamma\left(t_{i-1}\right)$ to $\gamma\left(t_{i}\right)$ (considered as points of $R^{2 k}$ ) within the set $L(K)$ by a sequence of $k$ moves such that for each $j=1,2, \cdots, k$, the $j$ th move carries the point $\left(b_{1}, b_{2}, \cdots, b_{j_{-1}}, a_{j}, \cdots, a_{k}\right)$ to the point $\left(b_{1}, \cdots\right.$, $\left.b_{j}, a_{j+1-1}, \cdots, a_{k}\right)$. Under the identification of $L(K)$ as an open subset of $R^{2 k}$, each of these $k$-moves clearly corresponds to a deformation from an element $g$ to some other element in $L(K)$ by moving a single vertex of $g(K)$. Hence, $\gamma\left(t_{i}\right)$ may be reached from $\gamma\left(t_{i-1}\right)$ by finitely many such single moves. This finishes the proof of Theorem $B$.

Theorem $B$ also allows an algebraic interpretation as follows: Let $D$ be a convex polygonal disk in $R^{2}$ and $\mathrm{PL}(D)$ be the set of all p.l. homeomorphisms of $D$ onto $D$. Observe that PL $(D)$ forms a group with respect to the composition of functions. For each triangulation $K$ of $D$, we let $S(K)=\{f \in L(K) \mid$ there is a vertex 
$v \in K$ such that $f \mid(D$-St $(v, K))=$ identity $\}$. It can be shown with the help of Remark 2.6 that for each triangulation $K$ of $D$, the set $S(K)$ consists of all elements of $L(K)$ which may be obtained by moving a single vertex of $K$. Now let $S(D)=\bigcup\{S(K) \mid K$ a triangulation of $D$. The set $S(D)$ may be called the set of single moves. Theorem B says that for each p.l. homeomorphism $f$ of $D$, there exists a triangulation $K$ of $D$ and a finite sequence of elements $f_{1}, f_{2}, \cdots$, $f_{m} \in S(D)$ such that

1. $f_{1} \in S(K)$ and $f_{i+1} \in S\left(f_{i}\left(\cdots\left(f_{1}(K)\right) \cdots\right)\right)$ for each $i=1, \cdots$, $m-1$.

2. $f=f_{m} \circ f_{m-1} \circ \cdots \circ f_{1}$.

In particular, each element of $\mathrm{PL}(D)$ is a finite product of elements of $S(D)$. Hence, we have the following.

THEOREM 4.4. For any convex polygonal disk $D$ in $R^{2}$, the group $\mathrm{PL}(D)$ is generated by the subset $S(D)$ of single moves.

\section{REFERENCES}

1. J. W. Alexander, On the deformation of an n-cell, Proc. Nat. Acad. Sci. U. S. A., 9 (1923), 406-407.

2. S. S. Cairns, Isotopic deformations of geodesic complexes on the 2-sphere and plane, Ann. of Math., 45 (1944), 207-217.

3. - Deformations of plane rectilinear complexes, Amer, Math. Monthly, 51 (1944), 247-252.

4. C. W. Ho, On certain homotopy properties of some spaces of linear and piecewise linear homeomorphisms I, Trans. Amer. Math. Soc., 181 (1973), 213-233.

5. - On certain homotopy properties of some spaces of linear and piecewise linear homeomorphisms II, Trans. Amer. Math. Soc., 181 (1973), 235-243.

6. J. F. P. Hudson, Piecewise Linear Topology, Math. Lecture Notes Series, W. A. Benjamin Inc., New York, 1969.

7. J. R. Munkres, Elementary Differential Topology, Ann. of Math. Studies, No. 54, Revised Ed., Princeton Univ. Press, 1966.

8. H. L. Smith, On continuous representations of a square upon itself, Ann. of Math., 19 (1917-18), 137-141.

9. H. Tietze, Über stetige Abbildungen einer Quädratflache auf sich selbst, Rend. Circ. Mat. Palermo, 38 (1914), 247-304.

10. O. Veblen, On the deformation of an n-cell, Proc. Nat. Acad. Sci., 3 (1917), 654659 .

11. J. H. C. Whitehead, On $C^{1}$-complexes, Ann. of Math., 41 (1940), 809-824.

Received February 26, 1974. Presented to the 79th Annual Meeting of the American Mathematical Society, January 25, 1973.

Southern Illinois University at EdWARdsville 



\section{PACIFIC JOURNAL OF MATHEMATICS}

\section{EDITORS}

RICHARD ARENS (Managing Editor)

University of California

Los Angeles, California 90024

\section{R. A. Beaumont \\ University of Washington \\ Seattle, Washington 98105}

\section{J. DugundJI}

Department of Mathematics

University of Southern California

Los Angeles, California 90007

D. Gilbarg and J. Milgram

Stanford University

Stanford, California 94305

\section{ASSOCIATE EDITORS}
E. F. BECKENBACH
B. H. NeumanN
F. WOLF
K. YOSHIDA

\section{SUPPORTING INSTITUTIONS}

UNIVERSITY OF BRITISH COLUMBIA
CALIFORNIA INSTITUTE OF TECHNOLOGY
UNIVERSITY OF CALIFORNIA
MONTANA STATE UNIVERSITY
UNIVERSITY OF NEVADA
NEW MEXICO STATE UNIVERSITY
OREGON STATE UNIVERSITY
UNIVERSITY OF OREGON
OSAKA UNIVERSITY

UNIVERSITY OF BRITISH COLUMBIA

UNIVERSITY OF CALIFORNIA

MONTANA STATE UNIVERSITY

NEW MEXICO STATE UNIVERSITY

OREGON STATE UNIVERSITY

OSAKA UNIVERSITY

\author{
UNIVERSITY OF SOUTHERN CALIFORNIA \\ STANFORD UNIVERSITY \\ UNIVERSITY OF TOKYO \\ UNIVERSITY OF UTAH \\ WASHINGTON STATE UNIVERSITY \\ UNIVERSITY OF WASHINGTON \\ AMERICAN MATHEMATICAL SOCIETY \\ NAVAL WEAPONS CENTER
}

The Supporting Institutions listed above contribute to the cost of publication of this Journal, but they are not owners or publishers and have no responsibility for its content or policies.

Mathematical papers intended for publication in the Pacific Journal of Mathematics should be in typed form or offset-reproduced, (not dittoed), double spaced with large margins. Underline Greek letters in red, German in green, and script in blue. The first paragraph or two must be capable of being used separately as a synopsis of the entire paper. Items of the bibliography should not be cited there unless absolutely necessary, in which case they must be identified by author and Journal, rather than by item number. Manuscripts, in triplicate, may be sent to any one of the editors. Please classify according to the scheme of Math. Reviews, Index to Vol. 39. All other communications should be addressed to the managing editor, or Elaine Barth, University of California, Los Angeles, California, 90024.

The Pacific Journal of Mathematics expects the author's institution to pay page charges, and reserves the right to delay publication for nonpayment of charges in case of financial emergency.

100 reprints are provided free for each article, only if page charges have been substantially paid. Additional copies may be obtained at cost in multiples of 50 .

The Pacific Journal of Mathematics is issued monthly as of January 1966. Regular subscription rate: $\$ 72.00$ a year (6 Vols., 12 issues). Special rate: $\$ 36.00$ a year to individual members of supporting institutions.

Subscriptions, orders for back numbers, and changes of address should be sent to Pacific Journal of Mathematics, 103 Highland Boulevard, Berkeley, California, 94708.

PUBLISHED BY PACIFIC JOURNAL OF MATHEMATICS, A NON-PROFIT CORPORATION

Printed at Kokusai Bunken Insatsusha (International Academic Printing Co., Ltd.), 270, 3-chome Totsuka-cho, Shinjuku-ku, Tokyo 160, Japan.

Copyright (C) 1973 by Pacific Journal of Mathematics Manufactured and first issued in Japan 


\section{Pacific Journal of Mathematics \\ Vol. 55, No. $2 \quad$ October, 1974}

Walter Allegretto, On the equivalence of two types of oscillation for elliptic

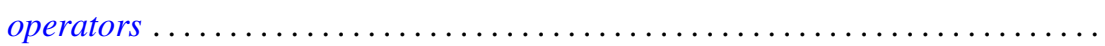

Edward Arthur Bertram, A density theorem on the number of conjugacy classes in

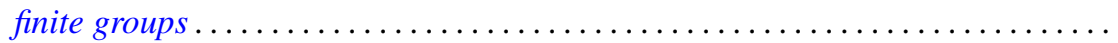

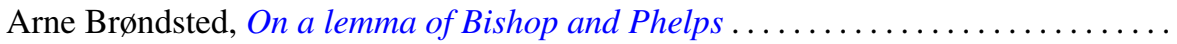

Jacob Burbea, Total positivity and reproducing kernels ..................

Ed Dubinsky, Linear Pincherle sequences . . . . . . . . . . . . . . . . . .

Benny Dan Evans, Cyclic amalgamations of residually finite groups .............

361

Barry J. Gardner and Patrick Noble Stewart, A "going down" theorem for certain

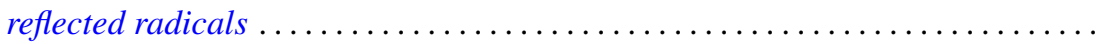

381

Jonathan Light Gross and Thomas William Tucker, Quotients of complete graphs:

revisiting the Heawood map-coloring problem ....................

Sav Roman Harasymiv, Groups of matrices acting on distribution spaces .........

Robert Winship Heath and David John Lutzer, Dugundji extension theorems for

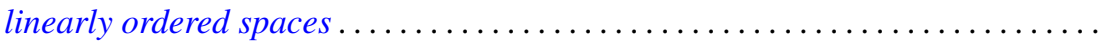

Chung-Wu Ho, Deforming p. l. homeomorphisms on a convex polygonal

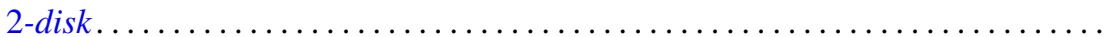

Richard Earl Hodel, Metrizability of topological spaces .................

Wilfried Imrich and Mark E. Watkins, On graphical regular representations of

cyclic extensions of groups .......................... 461

Jozef Krasinkiewicz, Remark on mappings not raising dimension of curves ..... . 479

Melven Robert Krom, Infinite games and special Baire space extensions . . . . . . 483

S. Leela, Stability of measure differential equations . . . . . . . . . . . . . . . . 489

M. H. Lim, Linear transformations on symmetric spaces . . . . . . . . . . . . . . . 499

Teng-Sun Liu, Arnoud C. M. van Rooij and Ju-Kwei Wang, On some group algebra modules related to Wiener's algebra $M_{1} \ldots \ldots \ldots \ldots \ldots \ldots \ldots \ldots \ldots \ldots \ldots$

Dale Wayne Myers, The back-and-forth isomorphism construction ............ 521

Donovan Harold Van Osdol, Extensions of sheaves of commutative algebras by

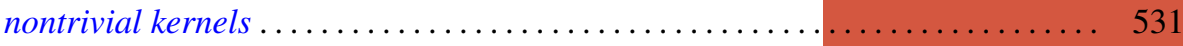

Alan Rahilly, Generalized Hall planes of even order ................... 543

Joylyn Newberry Reed, On completeness and semicompleteness of first countable

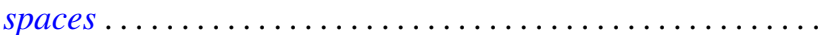

Alan Schwartz, Generalized convolutions and positive definite functions associated

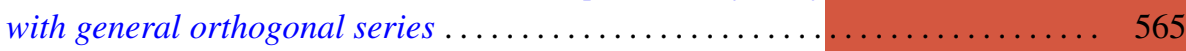

Thomas Jerome Scott, Monotonic permutations of chains . . . . . . . . . . . 583

Eivind Stensholt, An application of Steinberg's construction of twisted groups .... 595

Yasuji Takeuchi, On strongly radicial extensions . . . . ................. 619

William P. Ziemer, Some remarks on harmonic measure in space . . . . . . . . . . 629

John Grant, Corrections to: “Automorphisms definable by formulas” . . . . . . . . 639

Peter Michael Rosenthal, Corrections to: "On an inversion for the general

Mehler-Fock transform pair" ......................... 640

Carl Clifton Faith, Corrections to: "When are proper cyclics injective” . . . . . . 640 